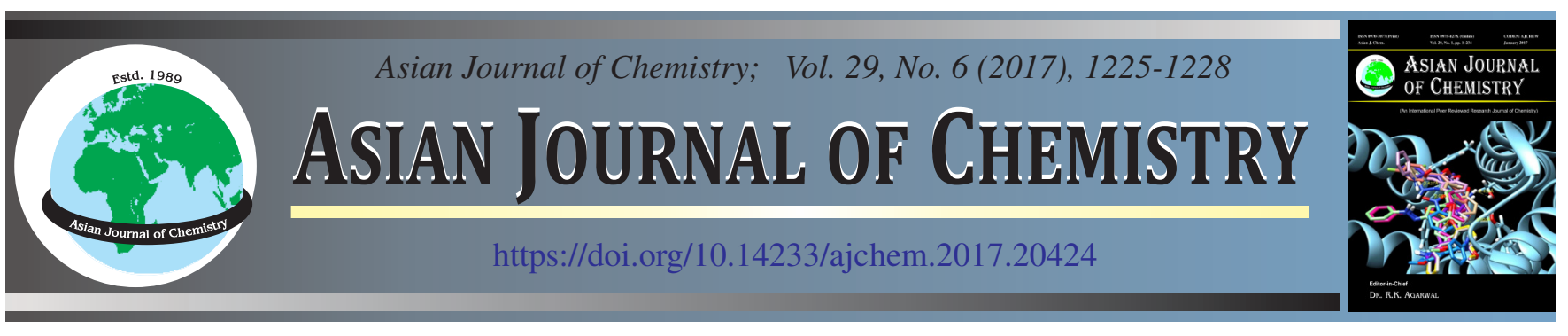

\title{
Energy Dependent Effective Atomic Number Calculation for Newly Developed 2-Acrylamido-2-methyl propane Sulfonic Acid Polymer Gel Dosimeter
}

\author{
P. SATHiYARAJ and E.J.J. SAMUEL ${ }^{*}$
}

Department of Physics and Medical Gel Dosimetry Laboratory, VIT University, Vellore-632 014, India

*Corresponding author: E-mail: ejames@ vit.ac.in

\begin{abstract}
The aim of the study is to evaluate the water equivalency of the newly developed polymer gel dosimeter [2-acrylamido-2-methyl propane sulfonic acid (AMPS)] in terms of its effective atomic number $\left(\mathrm{Z}_{\mathrm{eff}}\right)$ and effective electron density $\left(\mathrm{N}_{\mathrm{eff}}\right)$. Auto- $\mathrm{Z}_{\mathrm{eff}}$ computer program was used to calculate the $Z_{\text {eff }}$ for photon energies ranging from 0.01 to $1000 \mathrm{MeV}$. 2-Acrylamido-2-methyl propane sulfonic acid polymer gel shows the good water equivalency with less than $1.56 \%$ deviation from water over the energy range of 0.01 to $1000 \mathrm{MeV}$. Since 2-acrylamido-2-methyl propane sulfonic acid is less toxic and a very good water equivalent medium for photon energies from 0.01 to $1000 \mathrm{MeV}$ it can be employed in clinically. The study also reveals that it is a good alternative gel for poly acrylamide gelatin tetrakis hydroxyl phosphonium chloride (PAGAT) in terms of its less toxicity and water equivalency.
\end{abstract}

Keywords: 2-Acrylamido-2-methylpropane sulfonic acid, Effective atomic number, Electron density, Polymer gel.

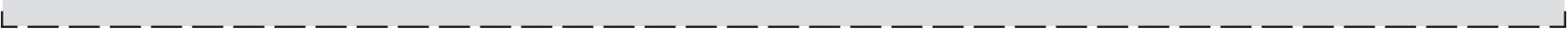

\section{INTRODUCTION}

Most of the polymer gel dosimeters are fabricated by radiosensitive monomers in gelatin matrix under normal atmospheric conditions [1-5]. Upon irradiation, free radicals are generated in the gel medium making the monomers active. Activated monomers form the polymer network. Absorbed dose of the radiation is correlated with the amount of polymerization by different readout modalities such as magnetic resonance imaging (MRI), optical computed tomography (OCT), X-ray computed tomography (X-ray CT), UV-visible spectrophotometer and ultra sound computed tomography (UCT) [6-10]. Since, water equivalency property is a major concern in radiation dosimeter, it is necessary to evaluate water equivalency for any medium which has to be used as an alternative for water. Effective atomic number $\left(\mathrm{Z}_{\text {eff }}\right)$ is the agreeable parameter to evaluate the water equivalency for a given medium [11]. The number that is introduced to describe the multi element material in terms of equivalent element is called the effective atomic number [12]. Most of the polymer gels are reported for good water equivalency $[13,14]$. Although, gel dosimeter has many advantages, its toxicity is making it inferior to other commercial dosimeters. Abtahi [15] introduced novel polymer gel with less toxic monomer by taking the well-known PAGAT formalism. In this formula acrylamide was replaced by 2-acrylamido2-methyl propane sulfonic acid (AMPS) and it is less toxic in comparison to acrylamide. With the introduction of new dosimeters (AMPS), different parameters were reported such as effective atomic number, toxicity and temporal stability. In the present study, the AMPS based polymer gel dosimeter's water equivalency based on its $\mathrm{Z}_{\text {eff }}$ was evaluated. Due to different photon interaction cross sections, different weights with respect to atomic composition, is given in the compound medium. Therefore a single number is not an appropriate way to express the $\mathrm{Z}_{\text {eff }}$ of the compound medium [16]. 2-Acrylamido-2methylpropane sulfonic acid gel dosimeter is an appreciable good gel dosimeter because of its low toxicity. To better establish its usability in clinical setting, the evaluation of $\mathrm{Z}_{\mathrm{eff}}$ with different photon energies shows its water equivalency. Eventually, it would encourage the researchers to improve its applicability clinically. The $\mathrm{Z}_{\text {eff }}$ calculation was performed for PMMA, soft tissue, poly acrylamide gelatin tetrakis hydroxyl phosphonium chloride (PAGAT) and water as well for comparative study with AMPS gel dosimeter.

\section{THEORETICAL ANALYSIS}

Effective atomic number $\left(\mathbf{Z}_{\text {eff }}\right)$ : Taylor et al. [17] developed the Auto- $Z_{\text {eff }}$ computer program to compute the $Z_{\text {eff }}$ for photon energies from $10 \mathrm{keV}$ to $1 \mathrm{GeV}$. In this computer program, photon interaction cross section matrices are constructed for the energy range of $10 \mathrm{KeV}$ to $1 \mathrm{GeV}$ and for the elements ranging between $Z=1$ to 100 . More details about the program are reported in the elsewhere [17]. Elemental compositions were obtained from XCOM database. $\mathrm{Z}_{\text {eff }}$ was 
calculated also for some radioactive sources such as $\mathrm{Co}^{60}, \operatorname{Ir}^{192}$, $\mathrm{Pd}^{103}$ and $\mathrm{Ra}^{226}$. The mean photon energies of radiation sources have been calculated using the following relation by taking the ratios of photons per disintegration from the radiation source [12].

$$
\mathrm{E}_{\mathrm{m}}=\frac{\sum_{\mathrm{i}} \mathrm{W}_{\mathrm{i}} \mathrm{E}_{\mathrm{i}}}{\sum_{\mathrm{i}} \mathrm{W}_{\mathrm{i}}}
$$

where $\mathrm{W}_{\mathrm{i}}$ is a branching ratio of each emitted photon of different energy, $E_{i}$ is the energy of the $i^{\text {th }}$ photon having the $W_{i}$ branching ratio.

Effective electron density $\left(\mathbf{N}_{\text {eff }}\right)$ : For an element electron density $\left(\mathrm{N}_{\text {eff }}\right)$ is given by the following equation:

$$
\mathrm{N}_{\mathrm{e}}=\frac{\mathrm{N}_{\mathrm{A}} \mathrm{Z}}{\mathrm{A}}
$$

But this expression cannot be used for multi elemental composition and it is altered by following relation.

$$
\mathrm{N}_{\text {eff }}=\mathrm{N}_{\mathrm{A}} \frac{\mathrm{nZ} \text { eff }}{(\mathrm{A})}
$$

where $\mathrm{A}$ is the average atomic mass of the material, $\mathrm{n}$ is the total number of atoms in the molecule and $\mathrm{N}_{\mathrm{A}}$ is the Avogadro number.

\section{RESULTS AND DISCUSSION}

The following Table-1 shows the mean photon energies for various $\gamma$-emitting sources.

\section{TABLE-1}

MEAN PHOTON ENERGIES FOR $\gamma$-EMITTING SOURCES

\begin{tabular}{cc}
\hline Radiation source & $\mathrm{E}_{\text {eff }}(\mathrm{MeV})$ \\
\hline $\mathrm{Pd}^{103}$ & 0.020 \\
$\mathrm{Ra}^{226}$ & 0.150 \\
$\mathrm{Ir}^{192}$ & 0.400 \\
$\mathrm{Co}^{60}$ & 1.253 \\
\hline
\end{tabular}

Effective atomic number and effective electro density: Fig. 1 shows the energy dependent effective atomic number of different water equivalent material. Initially $(0.01 \mathrm{MeV})$ all the compounds started with maximum value of $Z_{\text {eff. }}$ The energy range below $0.01 \mathrm{MeV}$ photoelectric absorption is the dominant process. Since, it is directly proportional to $\mathrm{Z}^{4-5}$ AMPS, PAGAT and soft tissue has slightly higher $Z_{\text {eff }}$ at this energy due to presence of high $\mathrm{Z}$ elements such as $\mathrm{P}, \mathrm{Cl}, \mathrm{S}, \mathrm{Na}$ and $\mathrm{K}$. Although water does not have any high $\mathrm{Z}$ component, it still has higher $Z_{\text {eff }}$ than PMMA at low energy region because of the presence of large proportion of oxygen in water. The reason for the rapid fall of $Z_{\text {eff }}$ in intermediate energy ( 0.01 to 0.1 $\mathrm{MeV}$ ), is that the photoelectric absorption does not dominate photon interaction after a few hundred keV. Compton cross section is directly proportional to $\mathrm{Z}$ and it makes $\mathrm{Z}_{\text {eff }}$ of all the compounds low and maintains constant value from 0.15 to 3 $\mathrm{MeV}$. From 3 to $150 \mathrm{MeV}$ the gradual increase was found since the process of pair production replaces the Compton process. Since, pair production is proportional to $\mathrm{Z}^{2}$, the $\mathrm{Z}_{\mathrm{eff}}$ was less than in photo electric absorption region. Beyond the $150 \mathrm{MeV}, \mathrm{Z}_{\mathrm{eff}}$ was maintained at a constant pattern. Since at very high energy range, pair production is the dominant process

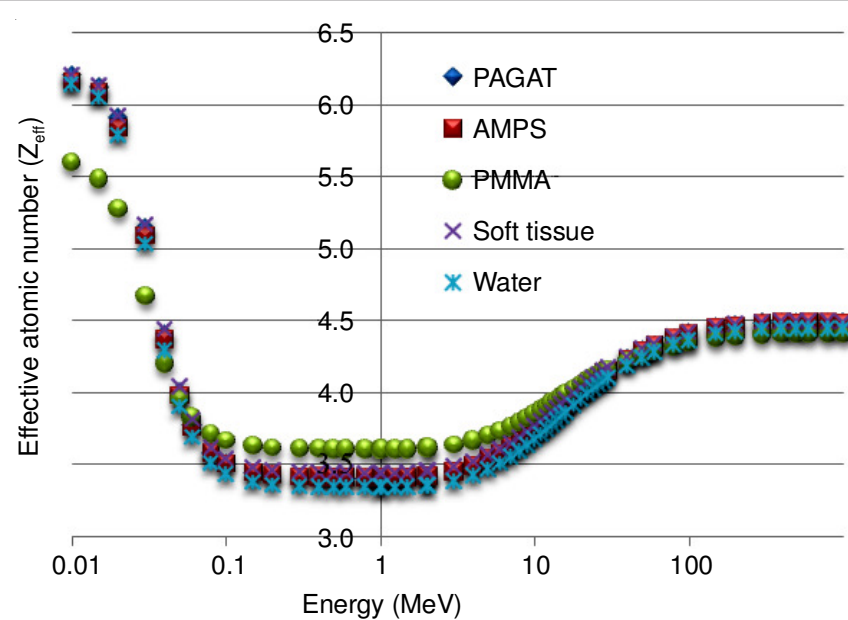

Fig. 1. Energy dependent effective atomic number for PAGAT, AMPS, PMMA, soft tissue and water

and it is proportional to $\mathrm{Z}$ of the material (beyond $150 \mathrm{MeV}$ ). Fig. 2 shows the $Z_{\text {eff }}$ of all the compounds for some $\gamma$-emitting radiation sources.

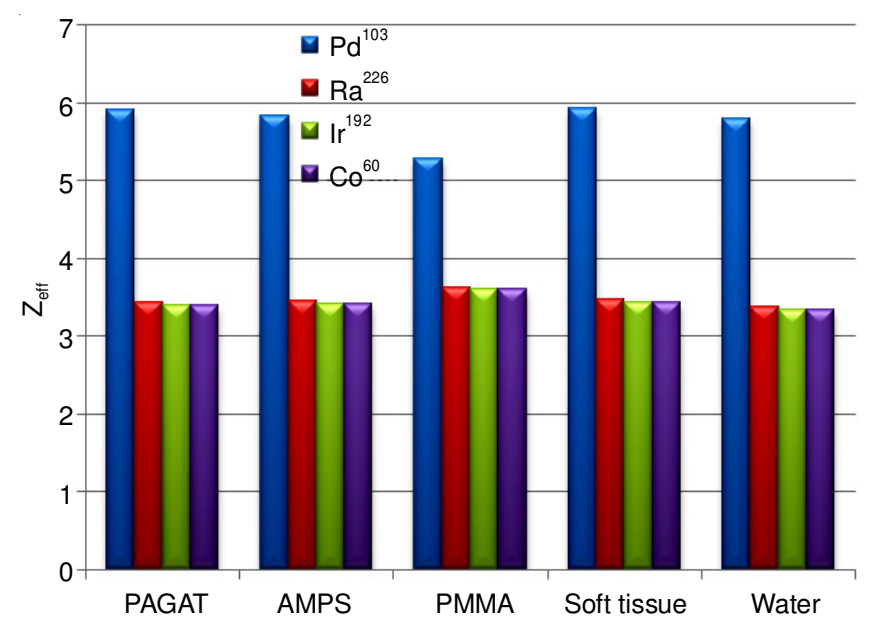

Fig. 2. Energy dependent effective atomic number of PAGAT, AMPS, PMMA, soft tissue and water for $\mathrm{Pd}^{103}, \mathrm{Ra}^{226}, \mathrm{Ir}^{192}$ and $\mathrm{Co}^{60}$

The effective energy of the $\mathrm{Pd}^{103}$ is less and the maximum interaction process could be attributed to the photoelectric absorption. This interaction process makes higher $\mathrm{Z}_{\mathrm{eff}}$ for all the compounds at the energy of Pd-103 source comparable with other radiation sources. Similar behaviour is observed for the values of $\mathrm{N}_{\mathrm{eff}}$ for all the analyzed compounds Fig. 3 . Water and soft tissue has higher $\mathrm{N}_{\text {eff }}$ than the other compounds since the average atomic number of both the compounds are less than the others.

Since $\mathrm{N}_{\mathrm{eff}}$ is derived from the value of $\mathrm{Z}_{\mathrm{eff}}$, it has a pattern similar to $Z_{\text {eff }}$ as seen in Fig. 1. The variation in $N_{\text {eff }}$ is slightly affected by the average atomic number of the compound. Fig. 4 shows the $\mathrm{N}_{\text {eff }}$ of all the compounds for different gamma emitting sources. Fig. 5 shows the strong co-relation between $\mathrm{N}_{\text {eff }}$ and $\mathrm{Z}_{\text {eff. }}$.

At low energies, the difference $\%$ of AMPS and PAGAT increases up to $2.5 \%$ (Fig. 6 Maximum percentage difference $(2.5 \%)$ was observed at $0.04 \mathrm{MeV}$ for PAGAT gel). From 0.05 to $2 \mathrm{MeV}$ the difference $\%$ of AMPS was less than $2.1 \%$. 


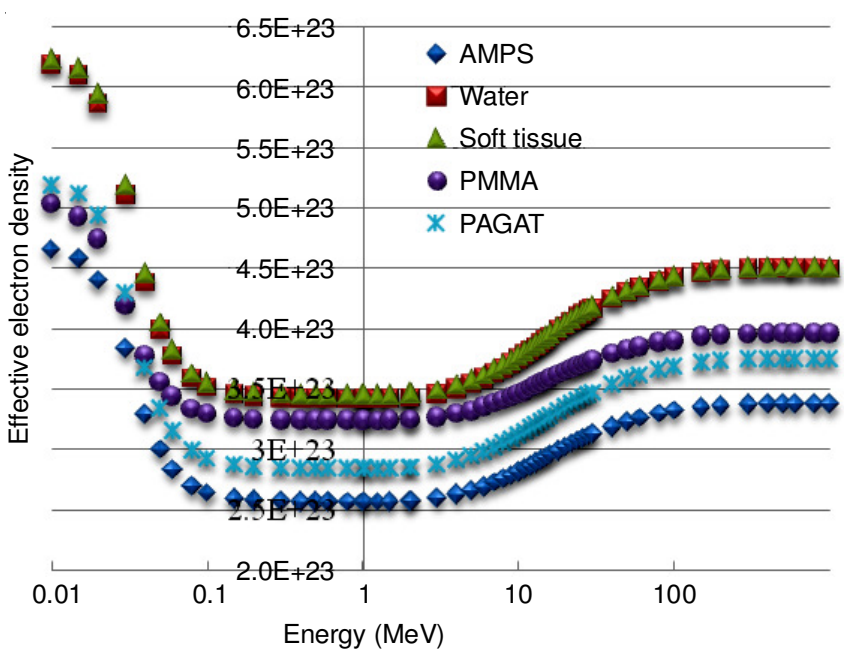

Fig. 3. Energy dependent effective electron density for PAGAT, AMPS, PMMA, soft tissue and water

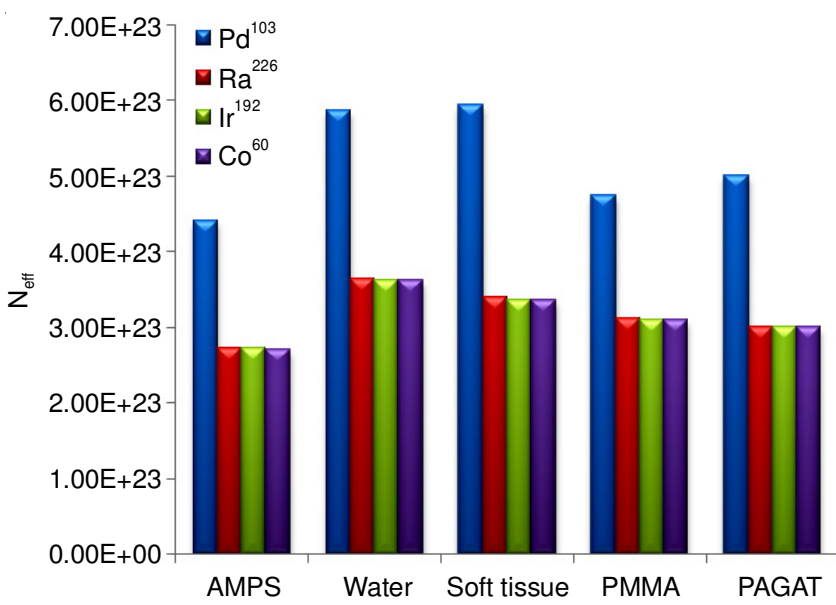

Fig. 4. Energy dependent effective electron density of PAGAT, AMPS, PMMA, soft tissue and water for $\mathrm{Pd}^{103}, \mathrm{Ra}^{226}, \mathrm{Ir}^{192}$ and $\mathrm{Co}^{60}$

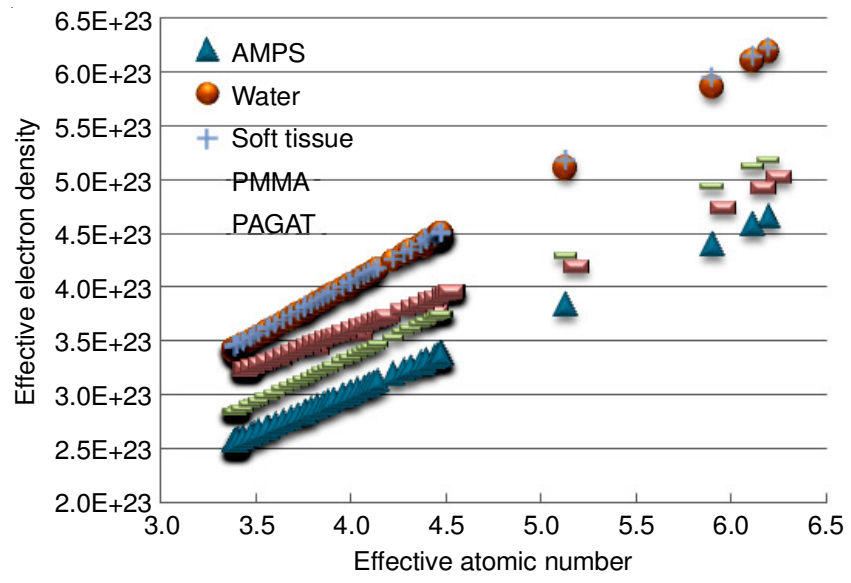

Fig. 5. Relation between Effective atomic number and electron density

The average percentage differences throughout the energy ( 0.01 to $1000 \mathrm{MeV}$ ) for AMPS and PAGAT are in between 1.56 and $1.54 \%$. The $Z_{\text {eff }}$ of AMPS was found to be little higher from the PAGAT. The higher concentration of gelatin, less concentration of water and the presence of sulfur may be the reasons for this variation.

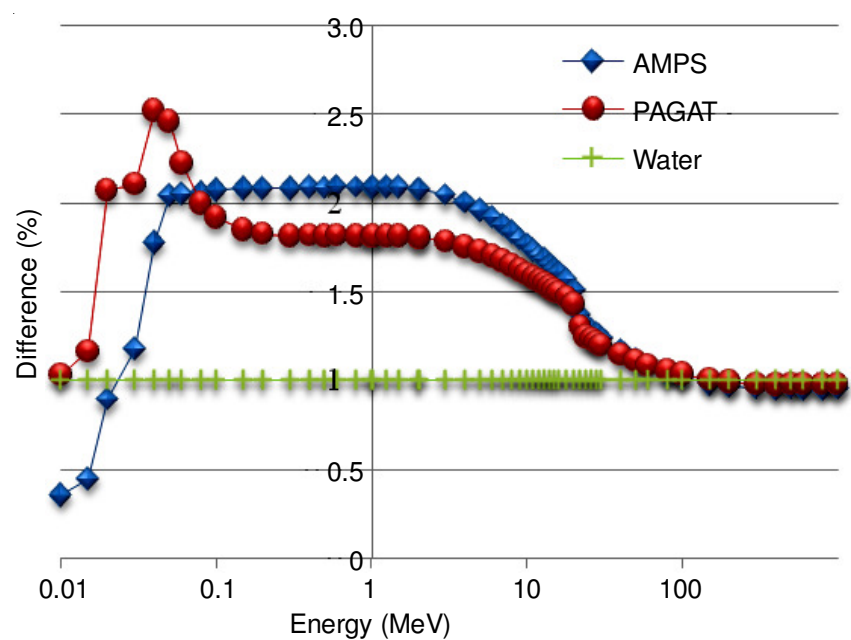

Fig. 6. Variation in the effective atomic number of AMPS and PAGAT with respect to water

Tetrakis(hydroxy methyl)phosphonium chloride (THPC) contains phosphorus and chlorine and AMPS contain sulfur. These elements have higher $\mathrm{Z}$ than hydrogen, carbon and nitrogen. Hence, the presence of THPC and AMPS increases the total mass attenuation co-efficient in the low energy region (0.01 to $0.08 \mathrm{MeV})$. Beyond that the impact of THPC and AMPS was found to be insignificant as seen in Fig. 7.

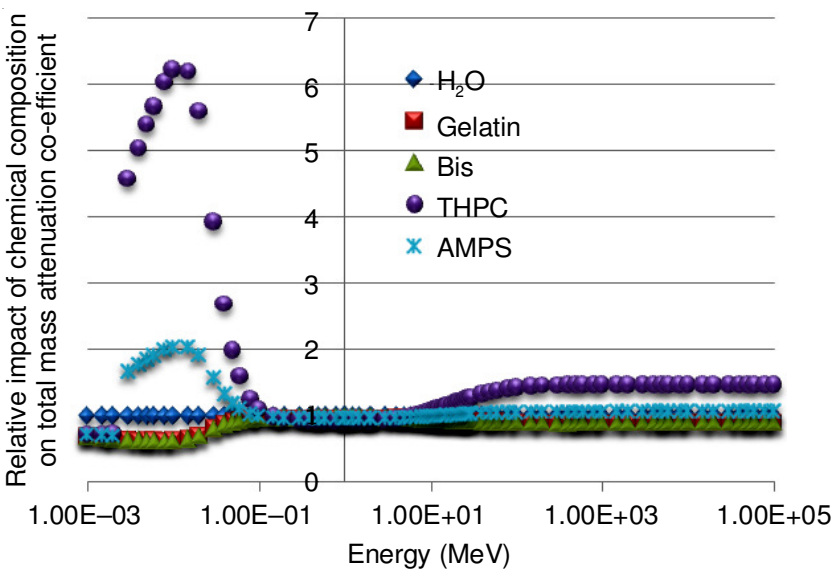

Fig. 7. Impact of chemical composition in energy absorption coefficient

\section{Conclusion}

Effective atomic number and effective electron density was successfully calculated for AMPS gel and the results were compared with existing phantom materials such as water, PMMA, soft tissue and PAGAT gel. Promising results were obtained for AMPS gel. The modification and introduction of new monomer in the well-known PAGAT formation has not affected the water equivalency of AMPS polymer gel significantly. Since AMPS is less toxic as well as a very good water equivalent medium from 0.01 to $1000 \mathrm{MeV}$, it can be employed in clinical applications. It is a good alternative gel for PAGAT in terms of its less toxicity and water equivalence property.

\section{ACKNOWLEDGEMENTS}

Dr. Gopishankar, Head of the Gamma knife unit, Department of Neuroscience, AIIMS, Delhi, India for technical support of the manuscript. 


\section{REFERENCES}

1. A.J. Venning, B. Hill, S. Brindha, B.J. Healy and C. Baldock, Phys. Med. Biol., 50, 3875 (2005); https://doi.org/10.1088/0031-9155/50/16/015.

2. A.J. Venning, K.N. Nitschke, P.J. Keall and C. Baldock, Med. Phys., 32, 1047 (2005);

https://doi.org/10.1118/1.1881812.

3. A. Hiroki, S. Yamashita, Y. Sato, N. Nagasawa and M. Taguchi, J. Phys. Conf. Ser., 444, 012028 (2013);

https://doi.org/10.1088/1742-6596/444/1/012028.

4. P. Sun, Y.C. Fu, J. Hu, N. Hao, W. Huang and B. Jiang, Radiat. Meas., 85, 116 (2016);

https://doi.org/10.1016/i.radmeas.2015.12.037.

5. T. Gorjiara, R. Hill, Z. Kuncic, S. Bosi, J.B. Davies and C. Baldock, Phys. Med. Biol., 56, 4685 (2011);

https://doi.org/10.1088/0031-9155/56/15/004.

6. M.J. Maryanski, R.J. Schulz, G.S. Ibbott, J.C. Gatenby, J. Xie, D. Horton and J.C. Gore, Phys. Med. Biol., 39, 1437 (1994); https://doi.org/10.1088/0031-9155/39/9/010.

7. M.J. Maryanski, Y.Z. Zastavker and J.C. Gore, Phys. Med. Biol., 41, 2705 (1996);

https://doi.org/10.1088/0031-9155/41/12/010.

8. M. Hilts, C. Audet, C. Duzenli and A. Jirasek, Phys. Med. Biol., 45, 2559 (2000);

https://doi.org/10.1088/0031-9155/45/9/309.
9. E.J.J. Samuel, P. Sathiyaraj, T. Deena and D.S. Kumar, J. Phys. Conf. Ser, 573, 012065 (2015); https://doi.org/10.1088/1742-6596/573/1/012065.

10. S. Khoei, J.V. Trapp and C.M. Langton, Phys. Med. Biol., 59, N129 (2014); https://doi.org/10.1088/0031-9155/59/15/N129.

11. T.K. Kumar and K.V. Reddy, Radiat. Phys. Chem., 50, 545 (1997); https://doi.org/10.1016/S0969-806X(97)00089-3.

12. M. Kurudirek, Radiat. Phys. Chem., 102, 139 (2014); https://doi.org/10.1016/j.radphyschem.2014.04.033.

13. P. Sellakumar, E. James Jebaseelan Samuel and S.S. Supe, Radiat. Phys. Chem., 76, 1108 (2007); https://doi.org/10.1016/i.radphyschem.2007.03.003

14. T. Gorjiara, R. Hill, Z. Kuncic, J. Adamovics, S. Bosi, J.H. Kim and C. Baldock, Med. Phys., 38, 2265 (2011); https://doi.org/10.1118/1.3561509.

15. S.M. Abtahi, Phys. Med., 32, 1156 (2016); https://doi.org/10.1016/j.ejmp.2016.08.008.

16. S.R. Manohara, S.M. Hanagodimath and L. Gerward, Phys. Med. Biol., 53, N377 (2008); https://doi.org/10.1088/0031-9155/53/20/N01.

17. M.L. Taylor, R.L. Smith, F. Dossing and R.D. Franich, Med. Phys., 39, 1769 (2012);

https://doi.org/10.1118/1.3689810. 